\title{
Rule Generation based on Reduct and Core: A Rough Set Approach
}

\author{
Renu Vashist \\ School of Computer Science and Engineering \\ Shri Mata Vaishno Devi University \\ Katra, (J \& K), India
}

\begin{abstract}
Rough set theory has evolved as one of the most important technique used for feature selection as a result of contemporary developments in data mining. One of the cardinal uses of Rough set theory is its application for rule generation. More often attribute reduction poses a major challenge for developing the theory and applications of rough set. This paper proposes a unique mathematical approach for determining the most important attribute with the help of confidence and strength of an association. Our approach focuses on the elimination of the redundant attributes in order to generate the effective reduct set (i.e., reduced set of necessary attributes) and formulating the core of the attribute set. Subsequently, only a subset of feature is selected which retain the accuracy of the original features.
\end{abstract}

\section{General Terms}

Artificial Intelligence, Data mining, Rough set, Decision tables.

\section{Keywords}

Rule generation, Rough set, Knowledge Discovery, Reduct, Core.

\section{INTRODUCTION}

Advent of internet and rising demand of business has resulted into many folds increase in the volume of data which is to be used for various applications on the day to day basis. This has posed an obvious challenge for anyone who needs to reduce the data set and find out the relevant data. This is where the rough set theory comes to the rescue in the form of an efficient tool for knowledge discovery in database(s). The ever growing field of Knowledge Discovery (KD) has been empowered from the developments of next generation databases and management information systems technology that helps in extraction of hidden useful information from the large databases[2][3]. This is aimed at formulation of knowledge for taking decisions by the organizations [1][4]. Data Mining is considered as an essential step in this knowledge discovery process and uses techniques from different disciplines, ranging from Machine Learning $[4][5][6][7], \quad$ Statistics $[4][5][6][7][8][9][10], \quad$ Information Sciences[4][5][6][7],Databases[7],Visualization[4][5][7][8][11],

\author{
Prof. M.L Garg \\ School of Computer Science and Engineering \\ Shri Mata Vaishno Devi University \\ Katra, (J \& K), India
}

Neural Networks [4][5][6][8][12], etc. Since Data Mining [11] borrows heavily the techniques from multiple disciplines therefore it has been regarded as a confluence of all these disciplines.

\section{BASIC CONCEPT OF ROUGH SET THEORY}

Rough set theory, introduced by Zdzislaw Pawlak in the early 1980 s is a new mathematical tool to deal with vagueness and uncertainty [2][3][4]. Rough set theory can be seen as a mathematical approach to intelligent data analysis and data mining.

Suppose, we are given an information system $S=(U, A), X \subseteq U$ UandP $\subseteq A$

where $\mathrm{U}$ and $\mathrm{A}$, are finite, nonempty sets and called as the universe, and the set of attributes, respectively. Set A will contain two disjoint sets of attributes, called condition and decision attributes and the system is donated by $S=(U, C, D)$ where $\mathrm{C}$ is called condition attribute and $\mathrm{D}$ is called decision attribute. With every attribute a $\epsilon$ A we associate a set $\mathrm{V}_{\mathrm{a}}$, of its values, called the domain of a.

Now we define two approximations $P(X)$ and $\overline{P(X)}$ called the $\mathrm{P}$-lower and the $\mathrm{P}$-upper approximation of $\mathrm{X}$ respectively where

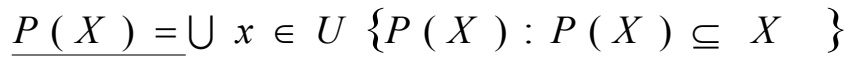

and

$\overline{P(X)=} \cup x \in U\{P(x): P(x) \cap X \neq \phi\}$

Lower approximation will consist of all the members which surely belongs to the set and Upper approximation consist of all the members which possibly belongs to the set. The boundary region is given by the set difference $\overline{P(X)}-P(X)$ consists of those objects that can neither be ruled in nor ruled out as members of the target set $X$. If the boundary region is 


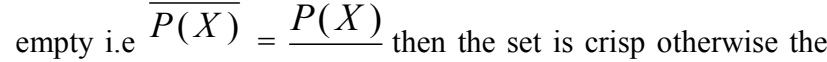
set is rough[2]. Rough set theory can determine whether there is any redundant information in the data and if it is there, then can we find essential data required for our applications.

The accuracy of the approximation to the set $\mathrm{X}$ from the elementary subsets is measured as the ratio of the lower and the upper approximation size. The ratio is equal to 1 , if no boundary region exists, which indicates a perfect classification. In this case, deterministic rules for the data classification can be generated. Thus, a set $\mathrm{X}$ with accuracy equal to 1 is crisp. otherwise $\mathrm{X}$ is rough[3].

\subsection{Reduct and Core}

Reduct and core are the two most important concept of rough set theory. Reduct is a reduced subset of original set which retains the accuracy of original set. Reduct is often used in the attribute selection process to reduce unnecessary attributes towards decision making applications[1].

1 Reduct of a decision table is a set of condition attributes that is sufficient to define the decision attribute.

2 Any reduct enables us to reduce condition attribute.

3 A reduct does not contain redundant attribute toward a classification task.

4 It reduces computation cost for rule generation.

5 Finding all the reduct is an NP-hard problem.

In a decision table we may find multiple reduct and some rule would appear more frequently in some reduct than others. There are so many methods of finding reduct of a decision table. The reducts can be obtained by using the reduct generation algorithms. Using the discernibility matrix, the reduct of a decision table can be found[1]. The core can be found as the set of all singleton entries in the discernibility matrix. The reduct is the minimal element in the discernibility matrix, which intersects all the element of the discernibility matrix.

\section{DATA REDUCTION AND FINDING THE RULES}

Rough sets have many applications in the field of Knowledge Discovery in Databases (KDD), such as feature selection, data reduction, discretization, etc. When a dataset contains irrelevant (dispensable) features the same may be eliminated and thereby reducing the dimension of the problem. Thereafter, Rough sets can be used to find subsets of relevant (indispensable) features [8].

The volume of data is increasing day by day. In many real applications, it is very difficult to find which attributes are important for a particular task and which attributes are not so important. Hence identifying the relevant features is important for the reduction of the volume of data. The aim of data reduction is to find a minimal subset of relevant attributes that have all the essential information of the data set, thus the minimal subset of the attributes can be used instead of the entire attributes set for rule discovery.

\subsection{Decision Table}

Rough set theory can be considered as an extension of classical set theory. The basic concept of the RST is the notion of approximation space, that is with every object of universe we associate some information i.e. Data and Knowledge. Every example of the Rough set is organized in the form of information table, whose columns are labeled as condition and decision attributes and rows of the table contain the example[3]. Entries in the table represents the attribute values.

Table 1 is a decision table whose decision attribute is $D$ and condition attributes are $\{\mathrm{x}, y, z, w\}$.

Table 1 Decision Table

\begin{tabular}{|c|c|c|c|c|c|}
\hline & \multicolumn{1}{|l|}{$\mathbf{y}$} & $\mathbf{z}$ & $\mathbf{\text { D }}$ \\
\hline 1 & $\mathrm{~A}$ & $\mathrm{P}$ & $\mathrm{A}$ & 3 & 1 \\
\hline 2 & $\mathrm{~A}$ & $\mathrm{P}$ & $\mathrm{S}$ & 1 & 1 \\
\hline 3 & $\mathrm{P}$ & $\mathrm{P}$ & $\mathrm{A}$ & 1 & 1 \\
\hline 4 & $\mathrm{P}$ & $\mathrm{R}$ & $\mathrm{A}$ & 3 & 2 \\
\hline 5 & $\mathrm{~A}$ & $\mathrm{R}$ & $\mathrm{A}$ & 2 & 2 \\
\hline 6 & $\mathrm{P}$ & $\mathrm{R}$ & $\mathrm{P}$ & 3 & 3 \\
\hline 7 & $\mathrm{~S}$ & $\mathrm{R}$ & $\mathrm{P}$ & 3 & 3 \\
\hline 8 & $\mathrm{~S}$ & $\mathrm{~N}$ & $\mathrm{P}$ & 3 & 3 \\
\hline 9 & $\mathrm{~S}$ & $\mathrm{~N}$ & $\mathrm{~S}$ & 2 & 2 \\
\hline 10 & $\mathrm{~S}$ & $\mathrm{~N}$ & $\mathrm{~S}$ & 2 & 1 \\
\hline
\end{tabular}

From Table 1 it is easy to see that for example 9 and 10 all the values of the condition attributes are same except for the values of decision attributes. We can say that Table1 is inconsistent because example 9 and 10 are conflicting (or are inconsistent) for both examples the value of all condition attribute is the same, yet the decision value is different. 


\subsection{Lower and Upper Approximations}

Rough set theory offers a tool to deal with inconsistencies[4]. For each concept $X$ the greatest definable set contained in $X$ and the least definable set containing $X$ are computed. The former set is called a lower approximation of $X$ the latter is called an upper approximation of $X$. In the case of Table 1, the elementary sets are $\{1\},\{2\},\{3\},\{4\},\{5\},\{6\},\{7\},\{8\},\{9,10\}$

Now, let us consider the concept for the table1. We can define decision attributes and elementary set associated with the decision- as subset of the set of all examples with the same value of decision. Such subset are called concept. There are three concepts in Table1.

$\mathrm{A} 1=\{1,2,3,10\}$ for decision 1

$\mathrm{A} 2=\{4,5,9\} \quad$ for decision 2

$\mathrm{A} 3=\{6,7,8\} \quad$ for decision 3

We can easily find lower and upper approximation of these three concepts.

$$
\underline{P(X)}=\{1,2,3,4,5,6,7,8\}
$$

Upper approximation is

$$
\overline{P(X)}=\{1,2,3,4,5,6,7,8,9,10\}
$$

The Boundary region is

Upper Approximation - Lower approximation $=\{9,10\}$

We are again drawing the consistent part of Table1. By removing conflicting example i.e. 9 and 10 .

Table2 Consistent Part Of Table1

\begin{tabular}{|l|l|l|l|l|l|}
\hline & $\mathbf{x}$ & $\mathbf{y}$ & $\mathbf{z}$ & $\mathbf{w}$ & $\mathbf{D}$ \\
\hline 1 & $\mathrm{~A}$ & $\mathrm{P}$ & 3 & $\mathrm{~A}$ & 1 \\
\hline 2 & $\mathrm{~A}$ & $\mathrm{P}$ & 1 & $\mathrm{~S}$ & 1 \\
\hline 3 & $\mathrm{P}$ & $\mathrm{P}$ & 1 & $\mathrm{~A}$ & 1 \\
\hline 4 & $\mathrm{P}$ & $\mathrm{R}$ & 3 & $\mathrm{~A}$ & 2 \\
\hline 5 & $\mathrm{~A}$ & $\mathrm{R}$ & 2 & $\mathrm{~A}$ & 2 \\
\hline 6 & $\mathrm{P}$ & $\mathrm{R}$ & 3 & $\mathrm{P}$ & 3 \\
\hline 7 & $\mathrm{~S}$ & $\mathrm{R}$ & 3 & $\mathrm{P}$ & 3 \\
\hline 8 & $\mathrm{~S}$ & $\mathrm{~N}$ & 3 & $\mathrm{P}$ & 3 \\
\hline
\end{tabular}

\subsection{Rule Generation}

Now we will generate the rules based on reduct and core of Table 2. Reduct is the reduced set of relation that conserves the same inductive classification of Relation. The set $P$ of attributes is the reduct (or covering) of another set $Q$ of attributes if $P$ is minimal and the indiscernibility relations, defined by $P$ and $\mathrm{Q}$ are same.

Core $=\cap$ reduct

Reduct of table 2 are $\{x, z, w\},\{x, y, w\},\{y, z, w\}$ and core of the table2 is attribute $w$. We can not eliminate attribute $w$ because this is the most important attribute of the Table2. By using the confidence or strength $(\alpha)$ we will find another indispensible attribute of the table. The confidence or strength for an association rule $\mathrm{x} \rightarrow \mathrm{D}$ is the ratio of number of example that contain $\mathrm{x} U \mathrm{D}$ to the number of example that contain $\mathrm{x}$.

For Table 2 we can calculate the strength of attribute $\mathrm{x}, \mathrm{y}$ and $\mathrm{z}$ as follows:

we can find the strength of rules for attribute $\mathrm{x}$

$(\mathrm{x}=\mathrm{A}) \rightarrow(\mathrm{D}=1)$ strength of this particular rule comes out to be $66 \%$.

$(\mathrm{x}=\mathrm{P}) \rightarrow(\mathrm{D}=1)$ strength of this particular rule comes out to be $33 \%$.

$(\mathrm{x}=\mathrm{P}) \rightarrow(\mathrm{D}=2)$ strength of this particular rule comes out to be $33 \%$.

$(\mathrm{x}=\mathrm{A}) \rightarrow(\mathrm{D}=2)$ strength of this particular rule comes out to be $33 \%$.

$(\mathrm{x}=\mathrm{P}) \rightarrow(\mathrm{D}=3)$ strength of this particular rule comes out to be $33 \%$.

$(\mathrm{x}=\mathrm{S}) \rightarrow(\mathrm{D}=3)$ strength of this particular rule comes out to be $100 \%$.

Similarly we can find the strength of rules for attribute $y$ and $\mathrm{z}$

$(y=P) \rightarrow(D=1)$ strength of this particular rule comes out to be $100 \%$.

$(y=R) \rightarrow \quad(D=2)$ strength of this particular rule comes out to be $50 \%$.

$(y=R) \rightarrow \quad(D=3)$ strength of this particular rule comes out to be $50 \%$.

$(y=N) \rightarrow \quad(D=3)$ strength of this particular rule comes out to be $100 \%$. 
and

$(\mathrm{z}=3) \rightarrow(\mathrm{D}=1)$ strength of this particular rule comes out to be $25 \%$.

$(\mathrm{z}=1) \rightarrow(\mathrm{D}=1)$ strength of this particular rule comes out to be $100 \%$.

$(\mathrm{z}=3) \rightarrow(\mathrm{D}=2)$ strength of this particular rule comes out to be $25 \%$.

$(\mathrm{z}=2) \rightarrow(\mathrm{D}=2)$ strength of this particular rule comes out to be $50 \%$.

$(\mathrm{z}=3) \rightarrow \quad(\mathrm{D}=3)$ strength of this particular rule comes out to be $100 \%$.

From these calculations we can easily find that attribute $y$ is indispensible among other attributes because the strength of rules for attribute $\mathrm{y}$ is maximum. The reduct of the set $\{\mathrm{x}, \mathrm{y}, \mathrm{z}, \mathrm{w}\}$ is $\{\mathrm{y}, \mathrm{w}\}$. Table 2 can be reduced to Table 3 as follows.

Table3

\begin{tabular}{|l|l|l|l|}
\hline & y & w & D \\
\hline 1 & P & A & 1 \\
\hline 2 & P & S & 1 \\
\hline 3 & P & A & 1 \\
\hline 4 & R & A & 2 \\
\hline 5 & R & A & 2 \\
\hline 6 & R & P & 3 \\
\hline 7 & R & P & 3 \\
\hline 8 & N & P & 3 \\
\hline
\end{tabular}

Reduce Table 3 by eliminating the same values of decision and condition attributes i.e we can merge different rows that has the same values for condition and decision attributes. This method is called Row Reduction.
Table 4

\begin{tabular}{|l|l|l|l|}
\hline & y & w & D \\
\hline 1 & P & A & 1 \\
\hline 2 & P & S & 1 \\
\hline 3 & R & A & 2 \\
\hline 4 & R & P & 3 \\
\hline 5 & N & P & 3 \\
\hline
\end{tabular}

Find out the core of each example

We will find the core of the Table 4 in such a manner that the table will remain consistent. If we eliminate $\mathrm{w}=\mathrm{A}$ there are two decision values 1 and 2 . It means that based on attribute $\mathrm{w}$ we cannot make a unique decision, thus the value of $y$ cannot be eliminated. Similarly if we eliminate $y=R$ there are two decision values 2 and 3 It means that based on attribute y we cannot make a unique decision, thus the value of $\mathrm{w}$ cannot be eliminated. Now table 4 becomes

Table 5

\begin{tabular}{|l|l|l|l|}
\hline & $\mathbf{y}$ & $\mathbf{W}$ & $\mathbf{D}$ \\
\hline 1 & $\mathrm{P}$ & $*$ & 1 \\
\hline 2 & $\mathrm{P}$ & $*$ & 1 \\
\hline 3 & $\mathrm{R}$ & $\mathrm{A}$ & 2 \\
\hline 4 & $*$ & $\mathrm{P}$ & 3 \\
\hline 5 & $*$ & $\mathrm{P}$ & 3 \\
\hline
\end{tabular}

Table 5 shows the core of each example. We can further reduced Table 5 by merging duplicate rows. Now we again eliminate the identical rows.

Table 6

\begin{tabular}{|l|l|l|l|}
\hline & $\mathbf{y}$ & $\mathbf{w}$ & D \\
\hline 1 & $\mathrm{P}$ & $*$ & 1 \\
\hline 2 & $\mathrm{R}$ & $\mathrm{A}$ & 2 \\
\hline 3 & $*$ & $\mathrm{P}$ & 3 \\
\hline
\end{tabular}


Now, no further reduction is possible. Table 6 gives us the decision rules. Followings are the decisions rules based on reduct and core:

$$
\begin{array}{llllll}
1 & \text { IF } \quad \mathrm{y} \rightarrow \mathrm{P} & & \text { THEN } & \mathrm{D} \rightarrow 1 \\
2 & \text { IF } \quad \mathrm{y} \rightarrow \text { R AND } \quad \mathrm{w} \rightarrow \mathrm{A} & \text { THEN } & \mathrm{D} \rightarrow 2 \\
& & & & & \\
3 & \text { IF } & \mathrm{w} \rightarrow \mathrm{P} & & \text { THEN } & \mathrm{D} \rightarrow 3
\end{array}
$$

\section{CONCLUSION}

This paper presents a new approach for determining the most important attribute on the basis of strength of an association. It is one of the most promising and new analytical approach of the Rough set theory that can be used for framing new decision rules. The application of this approach may be used extensively in the fields of knowledge discovery, data mining or any other field concerning attribute reduction and feature selection. As a direction for future research attempts may be made towards testing this method using some large databases and comparing this method with some others existing methods.

\section{REFERENCES}

[1] Pal S.K., Skowron (Eds.), A. 1999. Rough Fuzzy Hybridization: A new trend in decision making. SpringerVerlag, Berlin.

[2] Pawlak, Z. 1982. Rough sets, International Journal of Computer and Information Sciences 11: 341-356.

[3] Pawlak, Z. 1991. Rough Sets: Theoretical Aspects of Reasoning about Data, System Theory, Knowledge Engineering and Problem Solving, vol. 9, Kluwer Academic Publishers, Dordrecht, The Netherlands.
[4] Han, Jiawei., Kamber, Micheline. 2001. Data Mining: Concepts and Techniques. San Franciso CA, USA, Morgan Kaufmann Publishers.

[5] Ramakrishnan., Naren and Grama, Ananth Y. 1999. Data Mining: From Serendipity to Science. IEEE Computer, August 1999: 34-37.

[6] Williams, Graham J. and Simoff, Simeon J. 2006. Data Mining Theory, Methodology, Techniques, and Applications (Lecture Notes in Computer Science/ Lecture Notes in Artificial Intelligence), Springer.

[7] Hand, D.J., Mannila, H., \& Smyth, P. 2001. Principles of Data Mining. Cambridge, MA: MIT Press.

[8] Hand, D.J., Blunt, G., Kelly, M.G. \& Adams, N.M. 2000. Data mining for fun and profit. Statistical Science, 15, 111131.

[9] Glymour, C., Madigan, D., Pregibon, D., and Smyth, P. 1996. Statistical inference and data mining. Communications of the ACM, 39(11):35-41.

[10] Hastie, T., Tibshirani, R., \& Friedman, J.H. 2001. Elements of statistical learning: data mining, inference and prediction. New York: Springer Verlag.

[11] Lee, H. and Ong, H., 1996. Visualization support for data mining. IEEE Expert, 11(5):69-75.

[12] Lu, H., Setiono, R., and Liu, H. 1996. Effective data mining using neural networks. IEEE Transactions on Knowledge and Data Engineering, 8(6):957-961. 